\title{
PARADOXOS DA LÓGICA DEÔNTICA: INDÍCIOS DE UM EQUÍVOCO
}

Ricardo Tavares da Silva* ricardo.silva@campus.ul.pt

RESUMO De acordo com a teoria das funções de verdade, a verdadel falsidade de uma proposição é computável a partir da verdade/falsidade das suas proposições "internas": para cada proposição (composta) há uma função (a respectiva) entre valores de verdade. Aplicada a proposições modais, origina a semântica dos mundos possíveis e, aplicada a proposições normativas, origina uma semântica que reduz os conceitos normativos aos conceitos modais, a semântica modal. Esta redução fica posta em questão com a existência dos chamados 'paradoxos da lógica deôntica'. Estes não podem ser vistos como meras anomalias, mas devem ser vistos como indícios da incorreção da semântica modal. Em todos eles, a regra da necessitação, assim como o axioma da distribuição, "não batem certo". Não obstante, o cumprimento/incumprimento das normas (não a verdade/falsidade das proposições normativas) já é computável a partir da verdade/falsidade das proposições que expressam os estados de coisas devidos.

Palavras chave: Paradoxos da Lógica Deôntica. Semântica Modal. Necessidade Normativa. Ambiguidade. Tabelas de Cumprimento.

Abstract: According to the theory of truth-functions the truth/falsehood of a proposition is computable from the truth/falsehood of its "internal" propositions: for each (molecular) proposition there is a function (its own function) between truth values. Possible world semantics arises from the application of that theory to modal propositions; modal semantics - the reduction of normative concepts to modal concepts - arises from its application to normative

1 Universidade de Lisboa. Artigo recebido em 26/09/2016 e aprovado em 09/12/2016. 
propositions. But the 'paradoxes of deontic logic', as they are called, undermine such reduction: they cannot be seen as mere anomalies but instead as evidence for the wrongness of modal semantics. In all these paradoxes, the necessitation rule, as well as the distribution axiom, fail. Nevertheless, the fulfillment/nonfulfilment of norms (not the truth/falsehood of normative propositions) is computable from the truth/falsehood of the propositions which express the required states of affairs.

Keywords: Paradoxes of Deontic Logic. Modal Semantics. Normative Necessity. Ambiguity. Fulfillment Tables.

\section{Introdução}

A observação de certas anomalias tem acompanhado o desenvolvimento contemporâneo da lógica deôntica, caracterizada como uma extensão da lógica modal. Ao conjunto dessas anomalias deu-se a designação genérica de 'paradoxos da lógica deôntica'. Outras designações podem ser atribuídas. McNamara (2014), por exemplo, socorre-se da expressão 'challenges to standart deontic logic' e utiliza frequentemente o termo puzzle.

O surgimento de tais paradoxos é originado, regra geral, pelo processo seguinte: seleciona-se determinado teorema da lógica modal; aplica-se ao domínio normativo; o teorema resultante é incompatível com as nossas intuições sobre o "comportamento" das normas. Tem de haver verdade tanto nesses (agora) teoremas da lógica deôntica quanto nas nossas intuições normativas mas, simultaneamente, são incompatíveis: e isso é paradoxal. Em muitos casos, também é paradoxal, por si mesmo, o próprio teorema de lógica deôntica a que se chega ${ }^{1}$.

O diagnóstico, defendo, é incorreto: não estamos perante paradoxos, mas, sim, falácias. Mais especificamente, estamos perante exemplificações da falácia da ambiguidade. Isto é: o termo 'obrigação' pode ser definido como 'necessidade normativa' mas esta não deve ser interpretada em sentido modal mas, sim, em sentido condicional (ser obrigatório é ser condição necessária de algo). A incompatibilidade referida atrás não é anômala: as intuições estão a assinalar que algo está mal com a aplicação de teoremas da lógica modal ao domínio normativo.

1 Como referem Schotch e Jennings (1981, p. 151), "deontic logic, from its birth, has served as a rich source of paradoxes. By this we mean that it has yielded results in the form of theorems and valid inferences which run counter to our intuitions about the intended interpretation of 'ought'". 
O entendimento ortodoxo, que vê na lógica deôntica uma extensão da lógica modal, reduz os conceitos normativos - obrigação, proibição, nãoobrigação e permissão - aos conceitos modais - necessidade, impossibilidade, não-necessidade e possibilidade (veja-se em Von Wright, 1951, e Anderson, 1958; esta tradição remonta, pelo menos, a Leibniz). Trata-se da continuidade de uma visão vero-funcional das proposições e da lógica em geral: a verdade/ falsidade de uma proposição é computável a partir da verdade/falsidade das suas proposições "internas".

Assim, tal como a fórmula proposicional 'É necessariamente verdade que $p$ ' adquire valor de verdade a partir do input dado a $p$ (tendo em conta o domínio dos mundos possíveis acessíveis a partir do mundo no qual se efectua a avaliação - em regra, o atual - e não apenas este último ${ }^{2}$ ), a fórmula proposicional 'É obrigatório $p$ ' também adquirirá valor de verdade a partir do input dado a $p$ (tendo em conta o domínio dos mundos bons acessíveis a partir do mundo no qual se efetua a avaliação) ${ }^{3}$.

Esta última restrição ao domínio dos mundos bons é importante. Sem ela, axiomas que valem num sistema de lógica modal, como $\square p \rightarrow p$ e $\neg \diamond p \rightarrow \neg p$, não valem, desde logo, num sistema de lógica deôntica: as obrigações e proibições podem ser violadas. Isto é: mesmo que $p$ seja obrigatório, ainda assim pode não ser verdade que $p$; mesmo que $p$ seja proibido, ainda assim pode ser verdade que $p^{4}$.

Ora, de acordo com uma semântica de mundos bons ou perfeitos, se (e só se) $p$ for uma proposição verdadeira em cada um dos mundos bons alternativos ao mundo atual, então será obrigatório $p$ e, se (e só se) $p$ for uma proposição falsa em cada um dos mundos bons alternativos ao mundo atual, então será proibido $p$. Assim, já se pode inferir a verdade de $p$ num qualquer mundo bom a partir da necessidade de $p$ restrita aos mundos bons, tal como se pode inferir a falsidade de $p$ num qualquer mundo bom a partir a impossibilidade de $p$ restrita aos mundos bons 5 .

A restrição bloqueia um argumento que pode ser usado contra a semântica modal (contra a atribuição de significado modal aos termos normativos): o fato

2 As discussões em torno da natureza e pertinência desta relação de acessibilidade não vão ser tidas em consideração aqui.

3 Nas palavras de Wedghood (2006, p. 131), "many philosophers understand 'ought' as a propositional operator-that is, as a term whose semantic value is a function from an embedded proposition (which is indicated in the sentence in which 'ought' occurs) to a further proposition".

4 Ou: mesmo que $p$ seja verdadeira em todos os mundos possíveis, ainda assim pode não ser obrigatório $p$ e, mesmo que p não seja verdadeira em todos os mundos possíveis, ainda assim pode não ser proibido p. Como afirma Von Wright (1971, p. 159), "that which necessarily is the case is also as a matter of fact the case; but that which ought to be the case is far from being always actually the case". Estamos perante um corolário da tradicional distinção entre dever ser verdade e ser verdade (ou entre estado de coisas devido e estado de coisas existente).

5 Pode dar-se o caso de $\mathrm{p}$ não ser verdadeira no mundo atual e ser verdadeira em cada um dos mundos bons alternativos ou ser verdadeira no mundo atual e ser falsa em cada um dos mundos bons alternativos. 
de os axiomas $\square p \rightarrow p$ e $\neg \diamond p \rightarrow \neg p$ não valerem num sistema de lógica deôntica revela que a lógica deôntica não constitui uma extensão da lógica modal. Ela constitui a garantia, que a teoria das funções de verdade tem, de que esta se mantém válida também no domínio normativo.

Claro que pode questionar-se se a obrigação de $p$ implica mesmo a verdade de $p$ num qualquer mundo bom. Pode defender-se que, mesmo aceitando que, para ser obrigatório $p$, é necessário e suficiente que $p$ represente um estado de coisas bom e, portanto, que seja verdadeira em pelo menos um mundo possível no qual exista o bem, nada nos diz que tem de representar um estado de coisas necessariamente bom e, como tal, que tem de ser verdadeira em todos os mundos possíveis nos quais exista o bem ${ }^{6}$.

Porém, não obstante essa restrição, há algo mais que continua a não "bater certo" na redução modal dos conceitos normativos. Na aplicação de certos teoremas da lógica modal, onde se esperaria que a obrigação e a proibição se "comportassem" como a necessidade, elas não o fazem. Surgem paradoxos, diz-se: por um lado, trata-se a obrigação e a proibição como conceitos modais (este é o lado da aplicação dos teoremas) e, por outro lado, verifica-se que a natureza da normatividade é avessa a essa aplicação (este é o lado das nossas intuições acerca do "comportamento" das normas).

Se admitirmos que há um equívoco na redução modal assinalada, os paradoxos desaparecem: se não há expectativa de que obrigação e proibição se "comportem" como necessidade e impossibilidade, não há conflito algum com intuições normativas. A perspectiva muda: o normal será, precisamente, que os teoremas da lógica modal não se apliquem no domínio normativo.

Selecionando alguns dos "paradoxos" da lógica deôntica mais paradigmáticos, podemos ver isso mesmo.

\section{O paradoxo de Ross}

Constitui um teorema da lógica deôntica que, se é obrigatório $p$, então é obrigatório $p$ ou $q: \mathrm{Op} \rightarrow \mathrm{O}(p \vee q)$. Ross $(1941$, p. 41; 1971, p. 149) dá um exemplo que se tornou famoso: se é obrigatório enviar uma carta, então é obrigatório enviá-la ou queimá-la ${ }^{7}$. O problema é que isso é contraintuitivo: como é que uma obrigação implica sua facultatividade? Como é que, de uma obrigação só ser cumprida se existir o estado de coisas devido, se infere que a

Para além disso, a restrição parece injustificável (ou arbitrária; ou ad hoc): pode estipular-se que "p" se refere a uma verdade num mundo bom mas não é essa a interpretação expectável quando esse símbolo é utilizado, pois a leitura normal é a de uma verdade no mundo atual. Aliás: se não pudermos inferir uma verdade no mundo atual, de nada serve um tal axioma.

7 Em rigor, este não é o exemplo avançado por Ross, pois que este fala em "imperativos" (comandos, ordens) e em validade, e não em proposições normativas e em verdade. 
obrigação pode ser cumprida mesmo que não exista aquele estado de coisas (e sim outro qualquer)?

A introdução da disjunção (inclusiva) constitui uma das regras da lógica proposicional clássica: se $p$ é verdadeira, então podemos sempre acrescentar uma disjunção $\operatorname{com} q$, pois a verdade da disjunção está assegurada pela verdade de $p$ ( $q$ pode ser falsa). Atendendo ao significado de ' $\mathrm{V}$ ' (pelo menos um dos disjuntos é verdadeiro), basta que a premissa, que se supõe verdadeira, seja um dos disjuntos da disjunção introduzida para que esta seja, também, verdadeira.

E se, em vez de $p$, tivermos $\square p$ ? Se $p$ for necessariamente verdadeira, que disjunção podemos acrescentar? Bem, desde logo, de $\square p$ infere-se $\square p \vee$ $\square q$. Como é só com base no significado de ' $\mathrm{V}$ ' que a inferência se dá, pode introduzir-se uma disjunção com qualquer "tipo" de proposição. Assim também com obrigações: de $\mathrm{O} p$ infere-se $\mathrm{O} p \vee \mathrm{O} q$. Isto é: de ser obrigatório enviar uma carta, por exemplo, infere-se que é obrigatório enviar essa carta ou que é obrigatório queimá-la. Aqui, não há problema: $p$ continua a ser obrigatório; não se torna facultativo.

Mas se, em vez de apenas considerarmos o significado da disjunção, também considerarmos o significado dos disjuntos? Neste caso, se $p$ for necessariamente verdadeira, que disjunção podemos acrescentar? De $\square p$, infere-se $\square(p \vee q)$ : para a disjunção ser necessariamente verdadeira, basta que um dos disjuntos o seja (já o converso não é válido). Portanto, de uma proposição necessariamente verdadeira infere-se tanto a necessidade dos disjuntos como a necessidade da disjunção.

Substituamos ' $\square p$ ' por ' $p$ é verdadeira em todos os mundos possíveis' e ' $\square(p \vee q)$ ' por 'a disjunção entre $p$ e $q$ é verdadeira em todos os mundos possíveis'. A inferência fica clarificada. Em cada um dos mundos possíveis, $p$ é verdadeira e pode ser introduzida a sua disjunção com $q$ : no mundo possível $\mathrm{M}_{1}, p$ é verdadeira e pode ser introduzida a disjunção com $q$; no mundo possível $\mathrm{M}_{2}$, $p$ é verdadeira e pode ser introduzida a disjunção $\operatorname{com} q$; no mundo possível $\mathrm{M}_{\mathrm{n}}, p$ é verdadeira e pode ser introduzida a disjunção $\operatorname{com} q^{8}$.

O mesmo pode ser dito quanto a uma proposição expressando uma obrigação? Se for obrigatório $p$, pode introduzir-se a obrigação de uma disjunção na qual $p$ seja um dos disjuntos? Se a lógica deôntica constitui uma extensão da lógica modal, de $\mathrm{O} p$ deveria inferir-se $\mathrm{O}(p \vee q)$. Por que razão isso é contraintuitivo? Porque o significado de 'obrigação' não permite a inferência. E, assim sendo, temos um indício muito forte de que há uma discrepância entre o significado dos termos modais e o significado dos termos normativos.

8 A chamada "regra da necessitação" permite tratar como verdade necessária qualquer regra lógica (até a própria regra da necessitação). 
Primeiro, não se pode substituir ' $\mathrm{O} p$ ' por ' $p$ é verdadeira em todos os mundos possíveis': mesmo sendo verdade (no mundo atual) que é obrigatório $p, p$ pode ser falsa (no mundo atual ou num qualquer mundo alternativo). $\mathrm{E}$, portanto, de $\mathrm{O} p$ não se infere $\square(p \vee q)$, quando de $\square p$ se infere $\square(p \vee q)$ : como não se pode dizer que $p$ é verdadeira em todos os mundos possíveis, não se pode acrescentar a disjunção com $q$ em todos os mundos possíveis.

Segundo, ' $\mathrm{O}(p \vee q)$ ' também não é substituível por 'a disjunção entre $p$ e $q$ é verdadeira em todos os mundos possíveis': mesmo sendo verdade (no mundo atual) que é obrigatório $p$ ou $q$, esta disjunção pode ser falsa (no mundo atual ou num qualquer mundo alternativo). Assim, fica bloqueada qualquer hipótese de relacionar $\mathrm{O} p$ e $\mathrm{O}(p \vee q)$ com base no valor de verdade de $p$.

Pode dizer-se o mesmo por outras palavras. Tanto a regra da necessitação quanto o axioma da distribuição, ambos importados de sistemas de lógica modal, não podem valer num sistema de lógica deôntica: i) ' $p \rightarrow(p \vee q)$ ' não é substituível por ' $\mathrm{O}[p \rightarrow(p \vee q)]$ ', embora o seja por ' $\square[p \rightarrow(p \vee q)]$, simplesmente porque o significado de 'obrigação' não permite essa substituição; ii) de $\mathrm{O}[p \rightarrow(p \vee q)]$ não se pode inferir $\mathrm{O} p \rightarrow \mathrm{O}(p \vee q)$, embora de $\square[p \rightarrow(p$ $\vee q)$ ] se possa inferir $\square p \rightarrow \square(p \vee q)$, pela mesma razão de índole semântica ${ }^{9}$.

Então e se substituirmos ' $\mathrm{O} p$ ' por ' $p$ é verdadeira em todos os mundos bons' e ' $\mathrm{O}(p \vee q)$ ' por 'a disjunção entre $p$ e $q$ é verdadeira em todos os mundos bons'? Já pode haver inferência? O que acontece é o seguinte: embora de $p$ ser verdadeira em todos os mundos bons se possa inferir que a disjunção entre $p$ e $q$ é verdadeira em todos os mundos bons, continua a não ser possível inferir $\mathrm{O}(p \vee q)$ de $\mathrm{O} p$. A discrepância não desapareceu com a restricção à interpretação modal de 'O'.

Mas, se se negar a redução operada pela semântica modal, então não se tem de aceitar o teorema em questão da lógica modal como um teorema da lógica deôntica, tendo como corolário o desaparecimento do paradoxo de Ross. Deixase de ter um teorema incompatível com as nossas intuições normativas: esta incompatibilidade indicia que as obrigações não constituem funções de verdade.

\section{O segundo paradoxo de Ross}

Constitui, igualmente, um teorema da lógica deôntica (“decalcada" da lógica modal) que, se é obrigatório $p$ e $q$, então é obrigatório $p: \mathrm{O}(p \wedge q) \rightarrow \mathrm{O} p$. Ross (1941, p. 43), mais uma vez, avança com o exemplo da carta: se é obrigatório enviar uma carta e queimá-la, então é obrigatório enviar essa carta (tal 
como é obrigatório queimá-la). Novamente, isto é contraintuitivo: a conjunção de dois fatos pode ser obrigatória sem que cada um deles isoladamente o seja. Porém, inferir a obrigação de enviar a carta (ou a obrigação de queimá-la) a partir da obrigação conjunta de enviar a carta e queimá-la é mesmo o expectável, caso a lógica deôntica constitua uma extensão da lógica modal. O que pode estar a correr mal, mais uma vez?

Aqui, está em questão outra das regras da lógica proposicional clássica, a eliminação da conjunção: se é verdade que $p \wedge q$, então é verdade que $p$. Basicamente, a regra decorre da definição de ' $\wedge$ ': este símbolo atesta que duas proposições são ambas verdadeiras, o que só acontece se cada uma delas for isoladamente verdadeira. Por isso, atendendo somente ao significado de ' $\wedge$ ', também tanto será verdade que $(\square p \wedge \square q) \rightarrow \square p$ como será verdade que ( $\mathrm{O} p \wedge$ $\mathrm{O} q) \rightarrow \mathrm{O} p$ (por exemplo, pode inferir-se a obrigação de enviar a carta a partir da conjunção da obrigação de enviar a carta com a obrigação de a queimar).

E quanto a $\square(p \wedge q) \rightarrow \square p$ ? Este teorema da lógica modal também se afigura intocável: se é necessariamente verdade que $p$ e $q$, então qualquer uma dessas proposições é necessariamente verdadeira - pois só assim pode a conjunção ser necessariamente verdadeira. Aliás, ‘ $\square(p \wedge q)$ ' e ‘ $\square p \wedge$ ' $\square q$ ' têm o mesmo significado.

Substituamos ' $\square(p \wedge q)$ ' por ' $p \wedge q$ é verdade em todos os mundos possíveis’e ' $\square p$ ' por ' $p$ é verdadeira em todos os mundos possíveis'. Em cada um dos mundos possíveis, a conjunção de $p \operatorname{com} q$ é verdadeira e pode ser eliminada a conjunção (mais uma vez, pela regra da necessitação) para ficarmos só com a verdade de $p$ : no mundo possível $\mathrm{M}_{1}$, a conjunção de $p \operatorname{com} q$ é verdadeira e pode ser eliminada a conjunção, ficando só a verdade de $p$; no mundo possível $\mathrm{M}_{2}$, a conjunção de $p$ $\operatorname{com} q$ é verdadeira e pode ser eliminada a conjunção, ficando só a verdade de $p$; no mundo possível $\mathrm{M}_{\mathrm{n}}$, a conjunção de $p \operatorname{com} q$ é verdadeira e pode ser eliminada a conjunção, ficando só a verdade de $p$.

$\mathrm{O}$ mesmo pode ser dito quanto a ' $\mathrm{O}(p \wedge q)$ '? Seguindo as nossas intuições normativas, não: pode ser obrigatório $p \wedge q$ (no mundo actual) e uma tal conjunção ser falsa nalgum mundo possível (no mundo atual ou num qualquer mundo alternativo), fazendo com que $\square p$ seja falsa. Para além disso, $\mathrm{O} p$ não é o mesmo que $\square p$ : como já se disse, mesmo sendo verdade (no mundo atual) que é obrigatório $p, p$ pode ser falsa (no mundo atual ou num qualquer mundo alternativo). Não há como ligar (com garantia) a verdade de $p$ num caso com a verdade de $p$ no outro: não há como relacionar $\mathrm{O}(p \wedge q)$ e $\mathrm{O} p$ com base na verdade de $p$.

É a diferença de significado de 'O (obrigação)' face ao de ' $\square$ (necessidade)' que gera estes resultados. Dada essa diferença, também aqui, tanto a regra da necessitação quanto o axioma da distribuição não podem valer num sistema 
de lógica deôntica ${ }^{10}$. E é por isso que, embora ' $\square(p \wedge q)$ ' e ‘ $\square p \wedge$ ‘ $\square q$ ' tenham o mesmo significado, já ' $\mathrm{O}(p \wedge q)$ ' e ' $\mathrm{O} p \wedge \mathrm{O} q$ ' não têm o mesmo significado: uma obrigação conjunta (ser obrigatório a conjunção de duas proposições) não é uma conjunção de obrigações (cada proposição ser obrigatória isoladamente).

E se substituirmos ' $\mathrm{O}(p \wedge q)$ ' por 'a conjunção entre $p$ e $q$ é verdadeira em todos os mundos bons' e 'Op' por ' $p$ é verdadeira em todos os mundos bons'? Mais uma vez, embora de a conjunção entre $p$ e $q$ ser verdadeira em todos os mundos bons se possa inferir que $p$ é verdadeira em todos os mundos bons, continua a não ser possível inferir $\mathrm{O} p$ de $\mathrm{O}(p \wedge q)$. Não há isomorfismo entre lógica clássica/lógica modal e lógica deôntica.

Esta "rebeldia" no "comportamento" das obrigações indicia que o significado de 'obrigação' é diferente do de 'necessidade'; nomeadamente, se a teoria das funções de verdade dá corretamente conta do significado do último termo, não o dá do do primeiro. Ora, basta rejeitar a redução operada pela semântica modal para os termos normativos para que o segundo paradoxo de Ross desapareça (por desaparecer a expectativa de que a obrigação se "comporte" como a necessidade).

\section{0 paradoxo do bom samaritano}

Prior (1958) avançou com o seguinte paradoxo: $\neg \mathrm{P} p \rightarrow \neg \mathrm{P}(p \wedge q)$ - se não é permitido (se é proibido) $p$, então não é permitido (é proibido) a conjunção de $p$ e $q$. Com uma versão ligeiramente diferente do exemplo que apresentou: se for proibido atacar uma pessoa, então é proibido atacá-la e socorrê-la, o que é contraintuitivo, mais uma vez. O exemplo pode ser alterado que a estranheza manterse-á: como é que, de uma proibição isolada, se salta para uma proibição conjunta?

Já se viu que a eliminação da conjunção constitui uma das regras da lógica proposicional clássica. Também já se viu que, pela regra da necessitação, pode ser aposta a necessidade a uma tal verdade lógica. Façamos, agora, contraposição: de $\square[(p \wedge q) \rightarrow p]$ segue-se $\square[\neg p \rightarrow \neg(p \wedge q)]$. Pelo axioma da distribuição ficamos com $\square \neg p \rightarrow \square \neg(p \wedge q)$. Como ' $\square \neg$ ' é interdefinível com ' $\neg \diamond$ ', obtém-se $\checkmark \neg p \rightarrow \neg \nabla(p \wedge q)$ '.

Este é um teorema de lógica modal, que é (intuitivamente) correto: se é impossível $p$ ser verdadeira, então é impossível que a conjunção de $p$ e $q$ seja verdadeira - mesmo que $q$ seja necessariamente verdadeira, como $p$ não pode ser verdadeira, não há qualquer hipótese de $p$ e $q$ serem, em conjunto, verdadeiras. Em termos de mundos possíveis: se $p$ é falsa em todos os mundos

10 Como observa McNamara (2014), estamos perante elementos do conjunto de puzzles em torno da regra que permite que, de $\vdash(p \rightarrow q)$, se infira $\vdash(\mathrm{O} p \rightarrow \mathrm{O} q)$. 
possíveis, então não há sequer um mundo possível no qual $p$ e $q$ sejam ambas verdadeiras (mesmo que $q$ seja verdadeira em todos os mundos possíveis).

Mas não pode ser um teorema de lógica deôntica. O ponto fundamental é este: de $p$ não ser possível segue-se que $p$ é falso, mas de $p$ não ser permitido não se segue que $p$ é falso. Percorrendo todos os mundos acessíveis a partir do atual, podemos ver a verdade de $p$ em alguns deles, mesmo não sendo permitido $p$. Como tal, mesmo que $\neg p \rightarrow \neg(p \wedge q)$ seja verdade em todos os mundos possíveis, não há garantia de $p \wedge q$ não ser verdade em todos esses mundos: em cada um deles, a conjunção fica em aberto por a verdade de $p$ continuar em aberto. Isto, porque, novamente, significado de 'proibição' não se coaduna com uma computação vero-funcional.

Tal manter-se-á assim mesmo que se restrinja o domínio modal aos mundos bons: embora seja verdade que, de $p$ ser falsa em todos os mundos bons, se segue que a conjunção de $p$ e $q$ também é falsa em todos os mundos bons, continua a ser contraintuitivo que, de $\neg \mathrm{P} p$, se siga $\neg \mathrm{P}(p \wedge q)$. A interpretação restritiva não afasta o paradoxo. Isso só acontece se for rejeitada a pressuposição de raiz, a de que os conceitos normativos se reduzem aos conceitos modais.

\section{O paradoxo da aparente indiferenciação}

Tal como o paradoxo do bom samaritano é construído a partir da eliminação da conjunção (embora, em rigor, o teorema em questão seja equivalente a uma introdução da disjunção), pode ser construído, de modo análogo, um paradoxo a partir da introdução da disjunção (embora o teorema resultante seja equivalente a uma eliminação da conjunção). Usemos as regras da necessitação deôntica e da contraposição no esquema inferencial da introdução da disjunção: obtemos $\mathrm{O}[\neg(p \vee q) \rightarrow \neg p]$. Pelo axioma da distribuição, obtemos $\mathrm{O} \neg(p \vee q) \rightarrow \mathrm{O} \neg p$ e, pela interdefinibilidade entre ' $\mathrm{O} \neg$ ' $\mathrm{e}$ ' $\neg \mathrm{P}$ ', obtemos $\neg \mathrm{P}(p \vee q) \rightarrow \neg \mathrm{P} p$.

Ficamos, assim, com algo como, por exemplo, ser proibido decidir precipitadamente ou decidir pressionado (qualquer uma delas indiferentemente) implica ser proibido decidir precipitadamente (categoricamente). Isso é equivalente a dizer que ser obrigatório (em conjunção) não-decidir precipitadamente e nãodecidir pressionado implica ser obrigatório não-decidir precipitadamente, o que não é verdade: decidir sem precipitação é insuficiente para cumprir a primeira das obrigações, enquanto é suficiente para cumprir a segunda. Não se pode simplesmente esquecer a relevância (normativa) de (não-)decidir pressionado.

Se estiverem em questão conceitos modais, não há problema. Usando necessitação e contraposição no esquema inferencial da introdução da disjunção, obtemos $\square[\neg(p \vee q) \rightarrow \neg p]$; pelo axioma da distribuição, obtemos $\square \neg(p \vee q) \rightarrow$ $\square \neg p$; e, pela interdefinibilidade entre ' $\square \neg$ ' $\mathrm{e}$ ' $\neg \nabla$ ', obtemos $\neg \nabla(p \vee q) \rightarrow \neg \nabla p$. 
Se não é possível que pelo menos uma de duas proposições seja verdadeira, então não é possível que qualquer uma delas seja verdadeira. Isso é intuitivamente correto.

A discrepância indicia, mais uma vez, que os termos normativos não podem ser interpretados modalmente. Mesmo com a restrição aos mundos bons, a discrepância mantém-se: de $p \vee q$ ser falsa em todos os mundos bons segue-se que $p$ é falsa em todos os mundos bons, mas de $\neg \mathrm{P}(p \vee q)$ continua a não seguir-se $\neg \mathrm{P} p$. De novo, o paradoxo desaparece se for abandonada a semântica modal.

\section{O paradoxo de Chisholm}

O último paradoxo que se analisará comunga do problema dos anteriores: a subsunção dos conceitos normativos nos conceitos modais. Mas diverge um pouco na forma. Chisholm (1963) dá um exemplo. É obrigatório que determinada pessoa preste assistência aos seus vizinhos: $\mathrm{O} p$. É obrigatório que, se o fizer, então os avisa que vai assisti-los: $\mathrm{O}(p \rightarrow q)$. Se não o fizer, então é obrigatório não os avisar: $\neg p \rightarrow \mathrm{O} \neg q$. Essa pessoa não lhes presta assistência: $\neg p$.

Intuitivamente, esse conjunto de quatro proposições é consistente. Desde logo, é consistente com a natureza das obrigações (seguindo as intuições normativas) que a obrigação $\mathrm{O} p$ não seja cumprida, isto é, $\neg p$. Por modus ponens, de $\neg p \rightarrow \mathrm{O} \neg q$ e $\neg p$ obtemos $\mathrm{O} \neg q$. Assim, embora fosse inicialmente obrigatório prestar assistência aos vizinhos, uma vez que essa obrigação não foi cumprida, surge a obrigação derivada de não se avisar que se vai assisti-los.

Mas, se seguirmos princípios da lógica deôntica standard, nomeadamente, o axioma da distribuição, o mesmo conjunto de proposições é inconsistente. Assim, de $\mathrm{O}(p \rightarrow q)$ obtém-se $\mathrm{O} p \rightarrow \mathrm{O} q$ e, por modus ponens, dado $\mathrm{O} p$, obtém-se $\mathrm{O} q$. Ora, já se tinha obtido $\mathrm{O} \neg q$. Portanto, $\mathrm{O} q$ e $\mathrm{O} \neg q$ serão ambas verdadeiras, o que não é possível. Se, seguindo as intuições normativas, o conjunto é consistente e se, seguindo a semântica modal, o conjunto é inconsistente, então a semântica modal não está de acordo com as nossas intuições normativas.

Pode causar estranheza que a forma lógica da frase 'É obrigatório que, se o fizer, então os avisa que vai assisti-los' seja ' $\mathrm{O}(p \rightarrow q)$ '. Uma relação condicional não pode ser obrigatória; dir-se-á que só a frase seguinte, 'Se não o fizer, então é obrigatório não os avisar' e a respectiva forma lógica ' $\neg p \rightarrow$ $\mathrm{O} \neg q$ ', têm sentido. Assim, aquela primeira significa verdadeiramente 'Se os for assistir, então é obrigatório avisá-los', cuja forma lógica é ' $p \rightarrow \mathrm{O} q$ '. Isso muda alguma coisa?

Se adotarmos uma interpretação modal irrestrita de 'O (obrigação)', de $\mathrm{O} p$ obtém-se $p$ e, por modus ponens, voltamos a obter $\mathrm{O} q$, mantendo-se a inconsistência. Pior: obtém-se uma contradição ( $p$ e $\neg p$ ): a obrigação de $p$ é inviolável 
mas, simultaneamente, já foi violada. Aqui, torna-se especialmente saliente a incompatibilidade entre semântica modal e intuições normativas. Se adotarmos uma interpretação modal restrita de 'O (obrigação)', a inconsistência (a contradição) mantém-se, embora não no mundo atual, mas num qualquer mundo bom.

Para evitar as complicações hermenêuticas que o conjunto de frases levanta e pondo de lado o recurso a obrigações derivadas, que podem desviar-nos do ponto que se visa frisar, veja-se uma versão mais simples do mesmo paradoxo, com o seguinte conjunto de fórmulas proposicionais: $\mathrm{O} p ; p \rightarrow q ; \neg q$. Intuitivamente, este conjunto é consistente: a obrigação de $p$ pode ser incumprida e, como tal, não ser ativado o consequente de $p \rightarrow q$, permitindo $\neg q$. Na interpretação modal, não o é: da obrigação de $p$ segue-se $p$, o que ativa $q$, que está em contradição com $\neg q$.

$\mathrm{Ou}$, em alternativa, considere-se um conjunto ainda mais simples: $\mathrm{O} p ; \neg p$. De acordo com aquilo que intuímos ser a natureza das obrigações, estamos perante um conjunto consistente: as obrigações são violáveis (isto é: mesmo sendo verdade que $p$ é obrigatório, ainda assim pode $p$ ser falsa; não há incompatibilidade). De acordo com a semântica modal, o conjunto é inconsistente: de $\mathrm{O} p$ segue-se $p$, em contradição com $\neg p$.

Se adotarmos a restrição aos mundos bons, das duas, uma: ou estendemos a restrição a todas as proposições, pelo que de $\mathrm{O} p$ ( $p$ é verdadeira em todos os mundos bons) se segue $p$ (a sua verdade num mundo bom particular), em contradição com $\neg p$ ( $p$ é falsa nesse mesmo mundo bom); ou não o fazemos, e de $\mathrm{O} p$ ( $p$ é verdadeira em todos os mundos bons) não se segue $p$ ( $p$ é verdadeira no mundo atual) mas $p$ num mundo bom particular, o que permite $\neg p$ (que $p$ seja falsa no mundo atual). Nesta última hipótese, o conjunto é consistente. Mas não há justificação para a verdade/falsidade da mesma proposição estar, num caso $(\mathrm{O} p)$, restrita aos mundos bons e, no outro caso $(\neg p)$, não o estar ${ }^{11}$.

Se há uma regra da lógica proposicional clássica que está em questão no paradoxo de Chisholm, ela é a do modus ponens (lembrando, de $p$ e $p \rightarrow q$ obtemos $q$ ). Apliquemos a regra da necessitação às premissas de uma inferência desse tipo: obtemos $\square p$ e $\square(p \rightarrow q)$. Aplicando o axioma da distribuição à condicional, obtemos $\square p \rightarrow \square q$. Temos, assim, as condições para efetuar o modus ponens modal: de $\square p$ e $\square p \rightarrow \square q$ obtemos $\square q$. O problema é que este esquema inferencial já não é válido se substituirmos ‘ $\square$ (necessidade)' por 'O (obrigação)': de ser obrigatório assistir os vizinhos e de ser obrigatório avisá-los, caso

11 Não se pode interpretar ' $p$ ' de maneiras diferentes na mesma situação: se, em 'Op', se restringe o domínio considerado aos mundos bons, então, em ' $\neg$ ', também tem de se efetuar a mesma restrição e, como se disse, a inconsistência/contradição mantém-se; se, em ' $\neg$ ', não se restringe o domínio aos mundos bons, então, em 'Op', também não se pode efetuar essa restrição e, então, de Op já não se segue p (o que seria de esperar se a normatividade fosse uma espécie de modalidade). 
se vá assisti-los, não se segue que é obrigatório avisá-los que se vai assisti-los. Pode ser verdade que não se vai assisti-los e, por isso, pode ser obrigatório avisá-los que não se vai assisti-los.

Como se verifica, este foi exatamente o processo seguido nos paradoxos analisados anteriormente. Em todos eles, a regra da necessitação (e o axioma da distribuição) falha(m) quando aplicada(os) ao domínio normativo: sempre que se introduz 'O(obrigação)' de modo análogo à introdução de ' $\square$ (necessidade)', a regra lógica resultante, que é válida no domínio modal, deixa de o ser no domínio normativo. O paradoxo de Chisholm padece dos mesmos problemas.

\section{Vero-funcionalidade deôntica}

Admitamos, então, que a verdade/falsidade de uma proposição normativa não é computável a partir da verdade/falsidade das suas proposições "internas": a fórmula proposicional 'É obrigatório $p$ ' não adquire valor de verdade a partir do input dado a $p$. Nota: isto não é o mesmo que dizer que não há proposições normativas ou que não há verdade/falsidade no domínio normativo. Aliás, à partida, se se fala em lógica das normas, está a aceitar-se que também no domínio normativo há verdade/falsidade (quanto a esta questão, veja-se o chamado 'dilema de Jørgensen') ${ }^{12}$.

Ainda assim, há uma maneira de as proposições normativas serem verofuncionais. Em vez de ser a verdade/falsidade das próprias proposições normativas a depender da verdade/falsidade das suas proposições internas, é o seu cumprimento/incumprimento. Não há qualquer erro em dizer-se que a verdade/ falsidade de uma proposição que expressa um estado de coisas devido constitui a condição de cumprimento/incumprimento desse dever.

Desta maneira, torna-se possível clarificar o significado de 'cumprimento' através de tabelas de "verdade" deônticas, tal como se clarifica o significado das conectivas proposicionais através de tabelas de verdade. Serão, então, tabelas de cumprimento: o input continua a ser a verdade/falsidade das proposições internas, mas o output passa a ser o cumprimento/incumprimento das normas.

Tabela do cumprimento/incumprimento da obrigação

\begin{tabular}{|c|c|}
\hline $\boldsymbol{p}$ & $\boldsymbol{O p}$ \\
\hline $\mathrm{V}$ & $\mathrm{C}$ \\
\hline $\mathrm{F}$ & $\mathrm{I}$ \\
\hline
\end{tabular}

12 Há que distinguir o âmbito curto do âmbito longo da verdade/falsidade associada a normas: uma coisa é a verdade daquilo que é obrigatório (âmbito curto) e outra é a verdade da própria obrigação (âmbito longo). Pode ser evidente que ser um estado de coisas existente não é o mesmo que nem causa ou implica ser um estado de coisas devido (a teoria das funções de verdade aplicada às normas nega isso) sem ser evidente que ser obrigatório é incompatível com ser verdade que é obrigatório. 
O "comportamento" das tabelas de cumprimento é, mutatis mutandis, idêntico ao das tabelas de verdade. Desde logo, como se vê, tanto o princípio da bivalência (uma proposição tem, no mínimo, um de dois valores de verdade, o verdadeiro e o falso) como o princípio da não-contradição (uma proposição tem, no máximo, um desses valores de verdade) são respeitados: uma norma é cumprida ou incumprida (não há terceiro "valor de cumprimento") e não é cumprida e incumprida (só tem um "valor de cumprimento"). Depois, a cada tabela de verdade clássica corresponde uma tabela de cumprimento específica, exactamente com as mesmas condições de "verdade".

Tabela de cumprimento da conjunção

\begin{tabular}{|c|c|c|}
\hline $\boldsymbol{p} \boldsymbol{q}$ & $\boldsymbol{p} \wedge \boldsymbol{q}$ & $\boldsymbol{O}(\boldsymbol{p} \wedge \boldsymbol{q})$ \\
\hline V V & V & C \\
\hline V F & F & I \\
\hline F V & F & I \\
\hline F F & F & I \\
\hline
\end{tabular}

Tabela de cumprimento da disjunção

\begin{tabular}{|c|c|c|}
\hline $\boldsymbol{p} \boldsymbol{q}$ & $\boldsymbol{p} \vee \boldsymbol{q}$ & $\boldsymbol{O}(\boldsymbol{p} \vee \boldsymbol{q})$ \\
\hline $\mathrm{V} \mathrm{V}$ & $\mathrm{V}$ & $\mathrm{C}$ \\
\hline $\mathrm{V} \mathrm{F}$ & $\mathrm{V}$ & $\mathrm{C}$ \\
\hline $\mathrm{F} \mathrm{V}$ & $\mathrm{V}$ & $\mathrm{C}$ \\
\hline $\mathrm{F} \mathrm{F}$ & $\mathrm{F}$ & $\mathrm{I}$ \\
\hline
\end{tabular}

Tabela de cumprimento da implicação (material)

\begin{tabular}{|c|c|c|}
\hline $\boldsymbol{p} \boldsymbol{q}$ & $\boldsymbol{p} \rightarrow \boldsymbol{q}$ & $\boldsymbol{O}(\boldsymbol{p} \rightarrow \boldsymbol{q})$ \\
$\mathrm{V} \mathrm{V}$ & $\mathrm{V}$ & $\mathrm{C}$ \\
$\mathrm{V} \mathrm{F}$ & $\mathrm{F}$ & $\mathrm{I}$ \\
$\mathrm{F} \mathrm{V}$ & $\mathrm{V}$ & $\mathrm{C}$ \\
F F & $\mathrm{V}$ & $\mathrm{C}$ \\
\hline
\end{tabular}

Tabela de cumprimento da negação

\begin{tabular}{|c|c|c|}
\hline $\boldsymbol{p}$ & $\neg \boldsymbol{p}$ & $\boldsymbol{O} \neg \boldsymbol{p}$ \\
\hline $\mathrm{V}$ & $\mathrm{F}$ & $\mathrm{I}$ \\
\hline $\mathrm{F}$ & $\mathrm{V}$ & $\mathrm{C}$ \\
\hline
\end{tabular}


Até os paradoxos da implicação material possuem o seu correspondente deôntico: sempre que a antecedente da condicional obrigatória for falsa, a obrigação é cumprida ${ }^{13}$; sempre que a consequente for verdadeira, obrigação também é cumprida. Para além disso, veja-se como qualquer obrigação quanto a verdades é equivalente a uma proibição quanto a falsidades: por exemplo, a obrigação de $p \mathrm{e}$ $q$ serem ambas verdadeiras é equivalente à proibição de pelo menos uma delas ser falsa. Basta olhar para os casos de cumprimento para identificar a obrigação e os casos de incumprimento para identificar a proibição.

Também para as negações das conectivas há tabelas de verdade e, como tal, também há tabelas de cumprimento. Pelo que foi dito no parágrafo anterior, podemos interpretar estas tabelas de cumprimento como sendo respeitantes a proibições.

Tabela de cumprimento da negação da conjunção

\begin{tabular}{|c|c|c|}
\hline $\boldsymbol{p q}$ & $\neg(\boldsymbol{p} \wedge \boldsymbol{q})$ & $\boldsymbol{O} \neg(\boldsymbol{p} \wedge \boldsymbol{q})$ \\
\hline $\mathrm{V} \mathrm{V}$ & $\mathrm{F}$ & $\mathrm{I}$ \\
\hline $\mathrm{V} \mathrm{F}$ & $\mathrm{V}$ & $\mathrm{C}$ \\
\hline F V & $\mathrm{V}$ & $\mathrm{C}$ \\
\hline F F & $\mathrm{V}$ & $\mathrm{C}$ \\
\hline
\end{tabular}

Tabela de cumprimento da negação da disjunção

\begin{tabular}{|c|c|c|}
\hline $\boldsymbol{p} \boldsymbol{q}$ & $\neg(\boldsymbol{p} \vee \boldsymbol{q})$ & $\boldsymbol{O} \neg(\boldsymbol{p} \vee \boldsymbol{q})$ \\
\hline $\mathrm{V} \mathrm{V}$ & $\mathrm{F}$ & $\mathrm{I}$ \\
\hline $\mathrm{V} \mathrm{F}$ & $\mathrm{F}$ & $\mathrm{I}$ \\
\hline F V & F & I \\
\hline F F & V & C \\
\hline
\end{tabular}

Tabela de cumprimento da negação da implicação (material)

\begin{tabular}{|c|c|c|}
\hline $\boldsymbol{p ~ q}$ & $\neg(\boldsymbol{p} \rightarrow \boldsymbol{q})$ & $\neg \boldsymbol{O}(\boldsymbol{p} \rightarrow \boldsymbol{q})$ \\
\hline V V & F & I \\
\hline V F & V & C \\
\hline F V & F & I \\
\hline F F & F & I \\
\hline
\end{tabular}


Tabela de cumprimento da negação da negação

\begin{tabular}{|c|c|c|}
\hline $\boldsymbol{p}$ & $\neg(\neg \boldsymbol{p})$ & $\boldsymbol{O} \neg(\neg \boldsymbol{p})$ \\
\hline $\mathrm{V}$ & $\mathrm{V}$ & $\mathrm{C}$ \\
\hline $\mathrm{F}$ & $\mathrm{F}$ & $\mathrm{I}$ \\
\hline
\end{tabular}

\section{Teoremas sem paradoxos}

Este método das tabelas de cumprimento permite-nos verificar que as regras da lógica proposicional clássica que estão na base dos paradoxos da lógica deôntica também se aplicam no domínio normativo, quando efetuadas as devidas correções. Se, em vez de verdade e falsidade, falarmos em cumprimento e incumprimento, não há paradoxos mas regras válidas.

Por analogia com as regras da lógica proposicional clássica, a validade pode ser aferida da seguinte maneira: comparando duas funções de cumprimento (não de verdade, não é demais lembrar), sempre que numa delas há cumprimento, na outra também há. Se isso acontece nos dois sentidos, então a função de cumprimento é a mesma. Se há cumprimento numa delas e, na outra, tanto há cumprimento quanto incumprimento, então a inferência é inválida.

Vejamos o equivalente da introdução da disjunção. Se 'Op $\rightarrow \mathrm{O}(p \vee q)$ ' for interpretada como expressando uma relação entre proposições normativas, então expressa uma condicional falsa. Mas, se for interpretada como expressando uma relação entre cumprimentos de normas, então expressa uma condicional verdadeira: sempre que $\mathrm{O} p$ é cumprida, $\mathrm{O}(p \vee q)$ também é cumprida.

Introdução da disjunção deôntica

\begin{tabular}{|c|c|c|}
\hline $\boldsymbol{p} \boldsymbol{q}$ & $\boldsymbol{O p}$ & $\boldsymbol{O}(\boldsymbol{p} \vee \boldsymbol{q})$ \\
\hline V V & C & C \\
\hline V F & C & C \\
\hline F V & I & C \\
\hline F F & I & I \\
\hline
\end{tabular}

Percebe-se perfeitamente que, sendo a obrigação de $p$ cumprida, a obrigação indiferenciada de $p$ ou $q$ também é cumprida. Claro que podemos, adaptando o que Hare diz relativamente ao paradoxo de Ross (tal como nos informa o próprio Ross em Ross, 1971, p. 149, nota de rodapé 19), desconfiar da pertinência de uma tal inferência: que sentido tem inferir que uma obrigação foi cumprida por uma de duas maneiras, sem saber qual, do fato de uma obrigação 
ter sido categoricamente cumprida por uma dessas maneiras? Se já se sabe por qual dessas maneiras a obrigação foi cumprida, para que inferir que o foi por uma delas indeterminadamente?

Como observa Ross, há que distinguir a relevância lógica da relevância epistêmica: logicamente, nada há a apontar à relação em questão; epistemicamente, então pode dizer-se que nada se ganha com a inferência, sendo até, deste ponto de vista, absurda. Mas, como Hare salienta, isso já se passa na introdução da disjunção clássica: não é próprio da introdução da disjunção deôntica.

O problema é mais profundo: podemos perguntar, logo à partida, se faz sentido $p$ ser categoricamente obrigatório e, simultaneamente, ser facultativamente obrigatório. E, efetivamente, pode fazer sentido se estivermos a falar de obrigações pertencentes a diferentes domínios normativos (moral e jurídico, por exemplo) ou, dentro do mesmo domínio normativo, pertencentes a diferentes contextos ou situações. Neste caso, até epistemicamente se torna pertinente ou relevante efetuar uma tal inferência (passa-se a saber que se "matou dois coelhos de uma só cajadada").

Também no caso do equivalente da eliminação da conjunção, se ' $\mathrm{O}(p \wedge q)$ $\rightarrow \mathrm{O} p$ ’ for interpretada como expressando uma relação entre proposições normativas, então expressa uma condicional falsa, mas, se for interpretada como expressando uma relação entre cumprimentos de normas, então expressa uma condicional verdadeira: sempre que $\mathrm{O}(p \wedge q)$ é cumprida, $\mathrm{O} p$ também é cumprida.

Eliminação da conjunção deôntica

\begin{tabular}{|c|c|c|}
\hline $\boldsymbol{p q}$ & $\boldsymbol{O}(\boldsymbol{p} \wedge \boldsymbol{q})$ & $\boldsymbol{O p}$ \\
\hline VV & C & C \\
\hline VF & I & C \\
\hline FV & I & I \\
\hline FF & I & I \\
\hline
\end{tabular}

O paradoxo do bom samaritano pode ser "regenerado", obtendo-se uma inferência válida. Mais uma vez, basta que se passe da linguagem da verdade/falsidade para a linguagem do cumprimento/incumprimento. Assim, se se cumpre a proibição de atacar uma pessoa, então cumpre-se a proibição de a atacar e de a socorrer. Se se incumpre a proibição de atacar uma pessoa, então, ainda assim, pode cumprir-se a proibição de a atacar e de a socorrer.

Embora de ser proibido decidir precipitadamente ou decidir pressionado não se possa inferir ser proibido decidir precipitadamente, do cumprimento da proibição de decidir precipitadamente ou decidir pressionado pode inferir-se o cumprimento da proibição de decidir precipitadamente. 
Eliminação da negação da disjunção deôntica

\begin{tabular}{|c|c|c|}
\hline $\boldsymbol{p q}$ & $\boldsymbol{O} \neg(\boldsymbol{p} \vee \boldsymbol{q})$ & $\boldsymbol{O} \neg \boldsymbol{p}$ \\
\hline $\mathrm{VV}$ & $\mathrm{I}$ & $\mathrm{I}$ \\
\hline $\mathrm{VF}$ & $\mathrm{I}$ & $\mathrm{I}$ \\
\hline $\mathrm{FV}$ & $\mathrm{I}$ & $\mathrm{C}$ \\
\hline $\mathrm{FF}$ & $\mathrm{C}$ & $\mathrm{C}$ \\
\hline
\end{tabular}

Também o modus ponens clássico tem o seu equivalente deôntico. Não se trata da mera conversão de ' $[\square p \wedge(\square p \rightarrow \square q)] \rightarrow \square q$ ' para a linguagem normativa, que não funciona. Mais uma vez, tem que ver com cumprimentos: a inferência é válida sempre que o cumprimento de uma obrigação em conjunto com o cumprimento de uma segunda obrigação condicionada pela existência do estado de coisas exigido pela primeira é acompanhado pelo cumprimento de uma terceira obrigação que exige o estado de coisas exigido condicionalmente pela segunda.

Modus ponens deôntico

\begin{tabular}{|c|c|c|c|}
\hline $\boldsymbol{p q}$ & $\boldsymbol{O} \boldsymbol{p}$ & $\boldsymbol{O}(\boldsymbol{p} \rightarrow \boldsymbol{q})$ & $\boldsymbol{O q}$ \\
\hline VV & C & C & C \\
\hline VF & C & I & I \\
\hline FV & I & C & C \\
\hline FF & I & C & I \\
\hline
\end{tabular}

O fato de haver uma lógica proposicional deôntica de cumprimentos e não de verdades reforça a reivindicação de que a lógica deôntica não pode ser "decalcada" da lógica modal (supondo que esta pode ser "decalcada" da lógica proposicional clássica). A verdade das proposições normativas não depende da verdade das proposições contidas naquelas (que expressam os estados de coisas devidos ou indevidos); só o seu cumprimento depende.

\section{Referências}

ANDERSON, A. R. "The Formal Analysis of Normative Systems". New Haven: International Laboratory, Sociology Department, Yale University, 1956.

. "A Reduction of Deontic Logic to Alethic Modal Logic". Mind, v. 67, n. 265, pp. 100-103, 1958.

CHISHOLM, R. "Contrary-to-Duty Imperatives and Deontic Logic”. Analysis, 24, pp. 33-36, 1963.

DANIELSSON, S. “Taking Ross's Paradox Seriously. A Note on the Original Proble- 
ms of Deontic Logic". Theoria, 71, pp. 20-28, 2005.

GABBAY, D.; HORTY, J.; PARENT, X.; VAN DER MEYDEN, R.; VAN DER TORRE, L. (Eds.). "Handbook of Deontic Logic and Normative Systems". London: College Publications, 2013.

HANSEN, J. "Imperatives and Deontic Logic - On the Semantic Foundations of Deontic Logic". Universidade de Leipzig: Tese (Doutorado), 2008.

. "The Paradoxes of Deontic Logic: Alive and Kicking". Theoria, v. 72, Issue 3, pp. 221-232, 2006.

HANSEN, J.; PIGOZZI, G.; VAN DER TORRE, L. “Ten Philosophical Problems in Deontic Logic". Schloss Dagstuhl, Germany: Dagstuhl Seminar Proceedings, 2007 [Online]. Disponível em: <http://drops.dagstuhl. de/opus/volltexte/2007/941/pdf/07122.PigozziGabriella.Paper.941.pdf $>$. Acesso em: 11 dez. 2016.

HILPINEN, R. (Ed.) "Deontic Logic: Introductory and Systematic Readings”. Dordrecht: D. Reidel, 1971.

. "New Studies in Deontic Logic". Dordrecht: D. Reidel, 1981.

MCNAMARA, P. "Deontic Logic". In: The Stanford Encyclopedia of Philosophy (Winter 2014 Edition), Edward N. Zalta (Ed.), Disponível em: <http://plato.stanford. edu/archives/win2014/entries/logic-deontic/>.

MELDEN, A. I. (Ed.). "Essays in Moral Philosophy". Seattle: University of Washington Press, 1958.

PRIOR, A. N. "The Paradoxes of Derived Obligation.” Mind, v. 63, pp. 64-65, 1954.

. (1958). "Escapism: The Logical Basis of Ethics." In: MELDEN, A. I. (Ed.). "Essays in Moral Philosophy". Seattle: University of Washington Press, 1958. pp. 135-146.

ROSS, A. "Imperatives and Logic.” Theoria, 7, pp. 53-71, 1941.

. "Logica de las Normas". Tradução para o espanhol de Jose Hierro, Madrid: Editorial Tecnos, 1971.

SHAFER-LANDAU, R. (Ed.) "Oxford Studies in Metaethics - Vol. 1". Oxford: Clarendon Press, 2006.

SCHOTCH, P.; JENNINGS, R. "Non-Kripkean Deontic Logic". In: HILPINEN, R. (Ed.) "New Studies in Deontic Logic". Dordrecht: D. Reidel, 1981. pp. 149-165

VON WRIGHT, G. H. "Deontic Logic.” Mind, v. 60, pp. 1-15, 1951.

. "Deontic Logic and the Theory of Conditions'. In: HILPINEN, R. (Ed.)

"Deontic Logic: Introductory and Systematic Readings". Dordrecht: D. Reidel, 1971. pp. 159-178.

WEDGHOOD, R. "The Meaning of 'Ought"”. In: SHAFER-LANDAU, R. (Ed.) "Oxford Studies in Metaethics - Vol. 1". Oxford: Clarendon Press, 2006. pp. 127-161. 